\title{
Día Mundial de la Diabetes, un llamado de atención
}

Con la consigna Tomemos control de la diabetes iya!, la Federación Internacional de Diabetes lanza el 14 de noviembre en todo el mundo, la advertencia de que esta enfermedad, cual tsunami en salud, amenaza el bienestar de millones de personas. Cada año, desde 1991, en esta fecha se organiza una campaña universal para llamar la atención de personas y gobiernos sobre la importancia de esta dolencia que en 2000 afectó a 151 millones y en este año, a 285 millones de pacientes, es decir, el 6,6\% de la población adulta mundial. Para 2030 se estima que la población afectada ascienda a 438 millones, un incremento del $54 \%$.

La Federación es una organización sombrilla con cerca de 215 asociaciones nacionales en más de 160 países, dirigida a la comunidad diabética desde 1950, cuya importancia social motivó a la Organización de Naciones Unidas, el 20 de diciembre de 2006, a promulgar la Resolución 61/225 sobre diabetes, y después, en 2007, dio un respaldo extraordinario a la lucha contra esta enfermedad, al convertir el 14 de noviembre en el Día Oficial de la Salud. Su relevancia fue patente al incluirla en la Alianza Mundial de Enfermedades Crónicas no Trasmisibles, para presentar un frente común de lucha contra el cáncer, las enfermedades respiratorias, la obesidad y las enfermedades cardiovasculares, dedicando los mayores esfuerzos a la atención primaria comunitaria, su prevención y la educación de las personas afectadas, con la particularidad de que interesan principalmente a los países más pobres del mundo, con ingresos económicos bajos y medianos, disminuyendo su natural capacidad de defensa. Fue así como el Foro Económico Mundial de 2009, sobre la visión 
global de riesgos a la salud, las identificó "como uno de los riesgos más significativos que enfrentan las economías, sólo superados por la elevación del precio de los combustibles, el recorte de gastos por la globalización, el colapso de países con ventajas como China, etc.". Para estudiar y enfrentar esta situación, las Naciones Unidas decidieron realizar en septiembre de 2011, cumpliendo lo acordado en su Asamblea General, a pedido de los Estados Miembros de la Comunidad Caribeña (Caricom), la Cumbre Mundial de Enfermedades Crónicas No Trasmisibles, con la finalidad principal, apoyada por más de 100 naciones, de analizar y resolver en lo posible los factores que producen estas enfermedades que afectan a los países pobres y en vías de desarrollo.

La cuantificación de la magnitud del problema de la diabetes mundial es extraordinariamente preocupante y Costa Rica no escapa a este hecho. El asistente del director general de la Organización Mundial de la Salud, Ala Alwan, ha dicho que "la Diabetes tiene muchas caras pero muy pocas voces". Se espera que en estas reuniones surjan soluciones y no más lamentos, porque entre 8 y 14 millones de personas mueren prematuramente cada año en los países en desarrollo, por diabetes mellitus, y si no se enfrenta el problema con soluciones, estas cifras aumentarán a 17 millones en la próxima década, con el mayor crecimiento en los países pobres con ingresos económicos de bajos a medianos, principalmente en África, con el $27 \%$, y en la regiones al este del Mediterráneo, con un $25 \%$, estimándose que el $80 \%$ de la mortalidad por enfermedades crónicas no trasmisibles se presenta en estos países. No se incluyen las cifras para la Región de Centro y Suramérica, donde las muertes atribuibles a diabetes en personas de 20 a 79 años ocupan el 9,5\% en las mediciones más creíbles. 
Costa Rica muestra cifras que deben llamar la atención a las autoridades de Salud: en una población de 2.963.000 personas con edades que oscilan entre 20 y 79 años, la prevalencia de diabetes mellitus tipo 2 es del 8,8\% para 261.700 diabéticos, con un tasa de 1,3 por 100.000 habitantes, por año. En las mismas edades, el porcentaje de prevalencia de intolerancia a la glucosa, el estado precursor natural de esta enfermedad, es del 7,0 para 206.600 personas, lo que augura que el porcentaje global de diabetes se mantendrá en la cifra anotada, con el consecuente alto gasto presupuestario en su atención. Es interesante que en este reporte, consignado en el Atlas de Diabetes, editado por la Federación Internacional de Diabetes en 2009, los autores no dispusieron de información sobre la existencia de un Programa Nacional de Diabetes en nuestro país, siendo que hay excelente evidencia de que la diabetes tipo 2 puede prevenirse, o al menos retrasarse su inicio, cuando existe un plan coordinado de acción. La mayor parte de la comprobación de este hecho se produce en personas en alto riesgo. Así, las modificaciones en los estilos de vida e intervenciones farmacológicas pueden reducir la aparición de nuevos casos hasta en un $60 \%$, demostrándose que la pérdida de peso en las personas obesas, el aumento de su actividad física mediante ejercicio y la dieta saludable, son más efectivos que las intervenciones farmacológicas.

Es claro entonces que Costa Rica debe instrumentar y poner en ejecución, lo antes posible, un Plan Nacional de Atención al Diabético y a su Grupo Familiar. Existen estudios de prevalencia y análisis de complicaciones crónicas en relación con variables de asistencia médica originadas en el Sistema Nacional de Salud. El hecho favorecedor de que la 
Caja Costarricense de Seguro Social entregue medicamentos antidiabéticos a toda la población que se presenta a recibir atención médica, tranquiliza a las autoridades en Salud. Pero se ha descuidado la universalidad de la educación en diabetes, pilar fundamental de su tratamiento. Hay escarceos diseminados en el país, producto más de las inquietudes y preocupaciones del personal en Salud que atiende a los diabéticos, que de un programa nacional en ejecución. El Ministerio de Salud ha realizado encuestas esclarecedoras acerca de la nutrición y de la diabetes, pero es preciso que sus hallazgos se traduzcan en acciones concretas, en conjunto con la Caja Costarricense de Seguro Social.

Buscar y apoyarse en las políticas de prevención, tratamiento y rehabilitación que conjuntamente han elaborado la Organización Mundial de la Salud y la Federación Internacional de Diabetes, será un extraordinario paso positivo. Los recursos existen y es urgente utilizarlos, pues la diabetes está también aquí y amenaza el bienestar de nuestros habitantes y el Sistema de Salud, si no se le toma en cuenta. Está plenamente demostrado que la prevención de las complicaciones crónicas de esta afección, en términos económicos, cuesta menos que tratarlas, con el dolor humano y la incapacidad física y mental que también producen.

Finalmente, señalan la Organización Mundial de la Salud y la Federación Internacional de Diabetes -instituciones que llaman la atención mundial sobre este problema de salud cada 14 de noviembre-, el problema debe verse como un asunto del desarrollo global de las naciones, pues amenaza su prosperidad. El $70 \%$ de la prevalencia mundial de diabetes se encuentra en los países pobres. Debe considerarse también que el gasto en la atención de la salud de los diabéticos cuenta para el 11,6\% del 
presupuesto de todas las enfermedades en el mundo, y el costo en dólares de los Estados Unidos de Norteamérica para este año, alcanza la suma de 376 billones, que proyectados a 2030 serán 490 billones. Lo catastrófico es que se estima que el $80 \%$ de estas sumas se gastarán en los países considerados ricos por su producto interno bruto, y solo el $20 \%$ en los países pobres y en desarrollo, donde viven más del $70 \%$ de los diabéticos.

Este editorial ha sido abundante en cifras estadísticas, con la deliberada intención de sustentar nuestra preocupación y propiciar la reflexión al respecto.

Dr. Eric Mora Morales

Miembro de Honorario de la Academia Nacional de Medicina

\section{Bibliografía}

1.-Informes de la Federación Internacional de Diabetes sobre el progreso del Día Mundial de la Diabetes, 2010

2. Diabetes Voice. Perspectivas mundiales de la Diabetes. Revista de la Federación Internacional de Diabete. 2010; 55

3. Eric Mora Morales. Diabetes Mellitus en Costa Rica.2006

4. Consultado en: www.diabetesatlas.org/ IDF Diabetes Atlas, Fourth Edition, accedido 10 de noviembre de 2010

5. Consultado en: www.eatlas.idf.org, accedido 10 de noviembre de 2010 